Z Gerontol Geriat 2013 · 46:532-542

DOI 10.1007/s00391-012-0463-7

Eingegangen: 21. September 2012

Überarbeitet: 8. November 2012

Angenommen: 19. November 2012

Online publiziert: 10. März 2013

๑) Springer-Verlag Berlin Heidelberg 2013

A. Koppitz ${ }^{1}$ J. Dreizler ${ }^{1}$ H. Hediger ${ }^{1} \cdot$ J. Voss ${ }^{2} \cdot$ L. Imhof ${ }^{1}$

${ }^{1}$ Institut für Pflege, Zürcher Hochschule für angewandte Wissenschaften (ZHAW), Winterthur

2 School of Nursing, University of Washington

\title{
Betreuungsindex in Pflegeheimen
}

\section{Entwicklung und Validierung eines neuen Instruments zur Beurteilung von Betreuungsqualität in Pflegeheimen}

Anstrengungen unternommen, Messinstrumente zu identifizieren. Ziel dieser Studie war es deshalb, Betreuung zu definieren und ein geeignetes Instrument zur Beurteilung von Betreuungsqualität zu identifizieren.

\section{Entwicklung der Definition von Betreuung}

Bei der Pflege und Betreuung dieser Personen werden Bedürfnisse nach

- Geborgenheit,

- Zufriedenheit,

- Kontrolle über die Umwelt,

- sozialer Interaktion und Zugehörigkeit,

- Austausch,

- Anerkennung und

- Wertschätzung $[3,4,5,6,7,8,9]$.

als besonders relevant für ein würdevolles Altern betrachtet.

Die kantonalen Pflegegesetze, z. B. $\$ \$ 5$ und 12 des Pflegegesetzes im Kanton Zürich, unterscheiden den Begriff Pflege von dem Begriff Betreuung [10]. Diese Aufteilung folgt der Logik der Bedürfnishierarchie nach Maslow [11]. Das Erhalten und Sichern von körperlichen Funktionen hat hierbei die oberste Priorität für den Gesetzgeber $[12,13,14]$.

Wie Betreuung definiert und wie sie gemessen werden kann, hat der schweizerische Gesetzgeber weder festgelegt noch
Ausgehend von den Fragen

- „Wie wird Betreuung definiert?“

- „Welche charakteristischen Eigenschaften können identifiziert werden?" und

- „Wie kann Betreuung gemessen werden?"

wurde zwischen den Jahren 2000 und 2010 eine Literaturrecherche in den Datenbanken Medline, Cinahl und Cochrane Libary mit den Schlagworten „scale“, „quality of life" und „caring" durchgeführt. Es wurde nach Definitionen von Betreuung und Instrumenten zur Messung der Betreuungsqualität in der stationären Langzeitversorgung pflegebedürftiger Personen gesucht.

Die Literatur wurde im ersten Schritt von zwei unabhängig voneinander arbeitenden wissenschaftlichen Fachpersonen durch die oben genannten Fragen analysiert. Im zweiten Schritt wurde eine Konzeptanalyse nach Walker und Avant [15] in Anlehnung an Wilson [16] von der
Erstautorin und dem Letztautor durchgeführt. Deren Ergebnisse wurden mittels Delphi-Verfahren in vier Sitzungen mit dem interdisziplinär besetzten Projektteam ( $\mathrm{n}=10$, Heimmanagement, Küche, Hauswirtschaft, Administration, Pflege, Hausdienst) diskutiert und konsentiert. Ebenfalls wurde eine Abgrenzung zu den Konzepten von Lebens- und Pflegequalität vorgenommen.

Lebensqualität. Lebensqualität kann als das zentrale Leitkonstrukt verstanden werden, das für die Outcomemessung von Betreuung relevant ist [17]. Als Kriterien und Einflussfaktoren auf die Lebensqualität werden

- krankheitsbedingte körperliche Beschwerden,

- die psychische Verfassung,

- erkrankungsbedingte funktionale Einschränkungen und

- die Ausgestaltung zwischenmenschlicher Beziehungen

bezeichnet [18]. Damit wird deutlich, dass die Beurteilung von Lebensqualität durch eine Vielzahl von objektiven und subjektiven Komponenten beeinflusst wird, die mit den Lebensbedingungen und der individuellen Bewertung der Betroffenen zusammenhängen.

Pflegequalität. Die Bewertung der Lebensbedingungen pflegebedürftiger Per- 


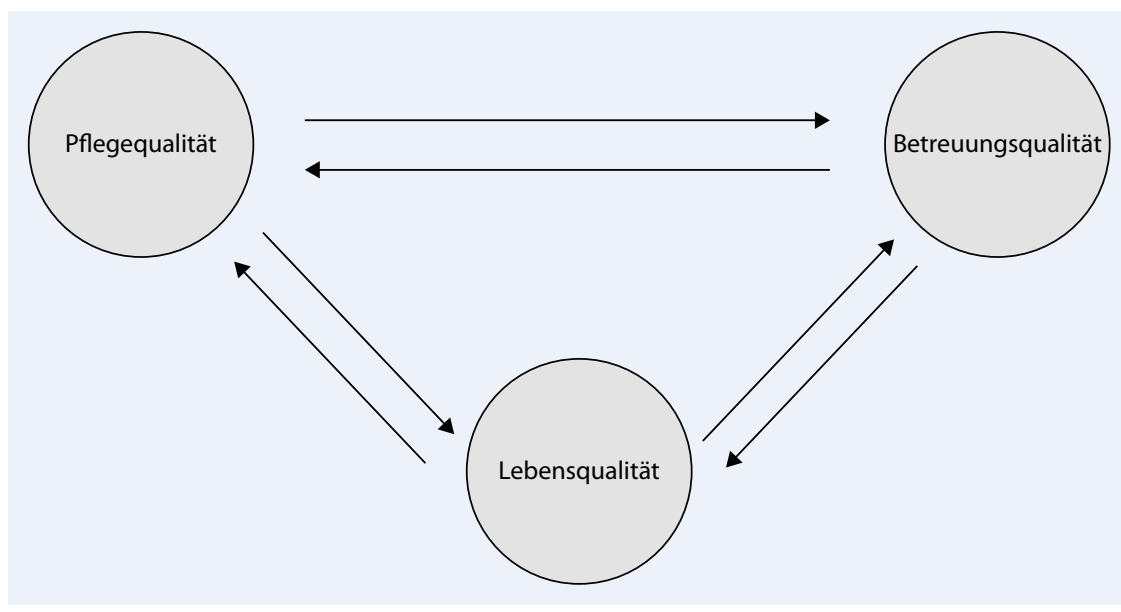

Abb. $1 \Delta$ Verhältnis von Lebens-, Pflege- und Betreuungsqualität

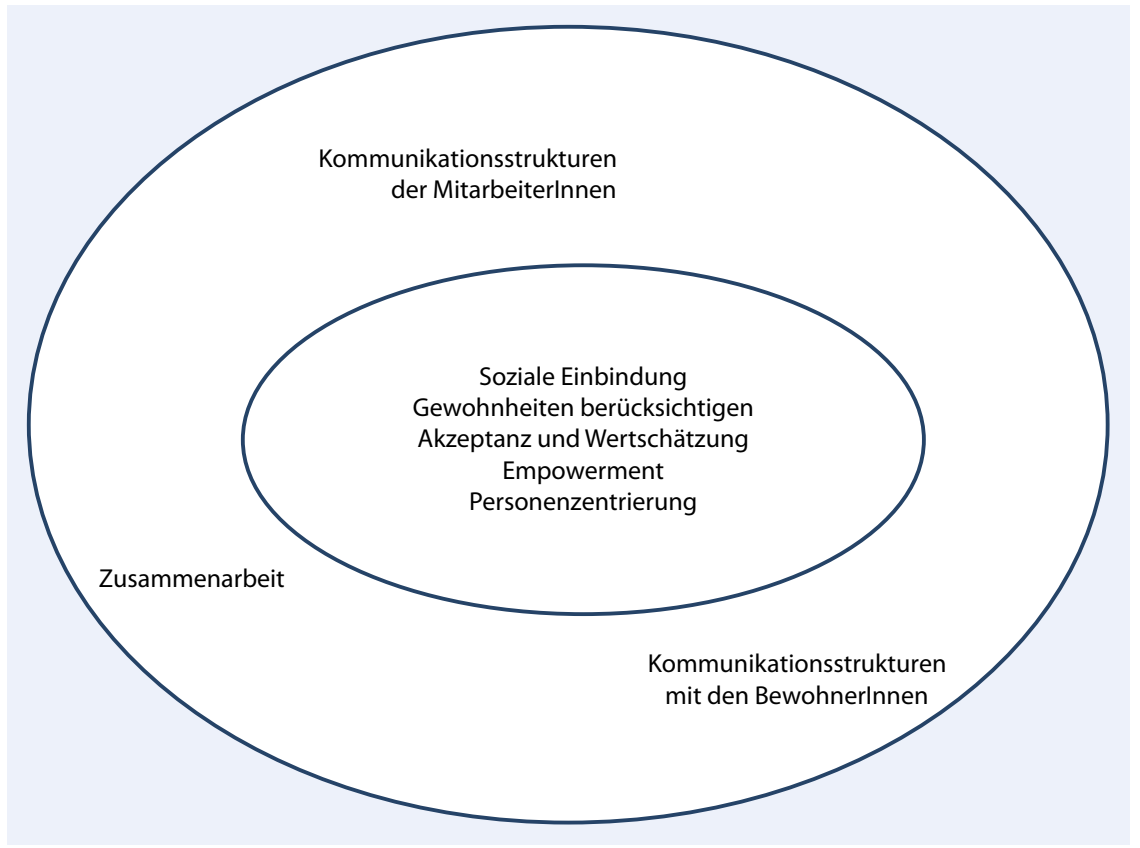

Abb. $2 \Delta$ Dimensionen von Betreuung

sonen in Pflegeheimen als objektive Komponente der Lebensqualität ist abhängig von der Pflegequalität $[19,20]$. Pflegequalität zeichnet sich aus insbesondere durch

- Erhaltung und Förderung von Selbständigkeit,

- dem Schutz vor Schädigungen und Belastungen und

- einer Unterstützung in spezifischen Bedarfslagen [20].

Caring. Die subjektive Komponente von Lebensqualität in Form der individuellen Bewertung hängt bei pflegebedürf- tigen Personen in Pflegeheimen mit Aspekten der Partizipation, Empowerment und der Bedeutsamkeit der Wahrnehmung der Betroffenen im Sinne der Personenzentrierung zusammen [19], die der Konzeption von "caring" entspricht. In - Abb. 1 soll der enge Zusammenhang von Lebens-, Pflege- und Betreuungsqualität verdeutlicht werden.

Der englische Begriff „,caring“, der im Deutschen mit „Betreuung“ übersetzt werden kann, betont die interpersonalen Kompetenzen der Pflegenden im Sinne von Sorge [21, 22, 23]. Bei der Sorge im pflegerischen Kontext geht es nicht um handlungsorientierte Aufzählung und Abarbeitung von Tätigkeiten. Vielmehr geht es um die menschliche Sorge gegenüber der pflegebedürftigen Person. Diese Art von Sorge beinhaltet beobachten, wahrnehmen, übersetzen, interpretieren, einschätzen, aushandeln, Vertrauen bilden, unterstützen und handeln [23]. Im Kontext der Pflege entspricht der deutschsprachige Begriff "Betreuung " als Synonym für "caring" der "pflegekundigen Sorge", der von Schnepp [24] geprägt wurde.

Betreuung. Als Ergebnis der eingangs erwähnten Konzeptanalyse konnten acht Konstrukte (Dimensionen) zur Betreuung identifiziert werden (• Abb. 2). Als Kernelemente von Betreuung wurden definiert - soziale Einbindung,

- Berücksichtigung von Gewohnheiten,

- Empowerment,

- Akzeptanz und Wertschätzung,

- Personenzentrierung,

- Zusammenarbeit und

- Kommunikationsstrukturen zwischen Mitarbeitern und Bewohnern.

Bei der sozialen Einbindung empfindet der Bewohner Art, Umfang und Inhalt der Betreuung so, wie er es sich wünscht. Der Bewohner erlebt zudem, dass seine Gewohnheiten im Heimalltag wichtig sind und Priorität genießen. Auch wird darauf geachtet, dass sich der Bewohner seine Fähigkeiten zur Entscheidung bis zuletzt erhält. Der Umgang mit Gefühlen, Haltungen und Meinungen wird von dem Bewohner als emphatisch wahrgenommen. Der Bewohner erlebt ein gelungenes Arrangement um seine Person. Der Austausch zwischen Bewohner und Mitarbeitern hat unmittelbaren Einfluss auf die Betreuung. Das gemeinsame Betrachten von Situationen steht im Mittelpunkt einer guten Betreuung.

Alle Mitarbeiter und Leitungspersonen sind gemeinsam für eine hohe Betreuungsqualität verantwortlich. Betreuung im Kontext der stationären Langzeitversorgung wurde deshalb definiert als sorgende Haltung und unterstützende Handlung, sowohl in Form von Art, Inhalt und Umfang von Programm- bzw. Beschäftigungsangeboten als auch als eine in den Alltag und in Abläufe integrierte Handlung. 
Betreuung verfolgt das Ziel

- der Einsamkeit vorzubeugen,

- erhält und sichert Gewohnheiten,

- befähigt die Person Entscheidungen zu fällen,

- vermittelt Akzeptanz und Wertschätzung und

- stellt die zu pflegende Person in den Mittelpunkt des Handelns.

Für das Verhältnis und die Abgrenzung von Lebens-, Pflege- und Betreuungsqualität in Pflegeheimen bedeutet dies, dass Pflege- und Betreuungsqualität einen zentralen Einfluss auf die Lebensqualität der pflegebedürftigen Personen hat [25, 26] und für die Outcome-Messung eine eigenständige und differenzierte Betrachtung von Pflege- und Betreuungsqualität notwendig erscheint.

\section{Vorhandene Messinstrumente für Betreuung}

Betreuungsqualität im Sinne der vorgestellten Definition zeichnet sich durch eine sorgende Haltung und konkreten Handlungen gegenüber den pflegebedürftigen Personen aus, die durch die acht Betreuungsdimensionen ( $\bullet$ Abb. 2) operationalisiert werden kann.

Allen im Rahmen der Literaturrecherche identifizierten 105 Instrumenten zur Messung von Betreuung ist gemeinsam, dass sie entweder eine krankheitsspezifische Ausrichtung haben oder den Anspruch erheben Lebensqualität umfassend betrachten und beurteilen zu wollen. Der Nachweis ausreichender Validität fehlt oft. Die Stichproben sind zu klein, um die Reliabilität der Instrumente zu beurteilen.

Die in der Literatur identifizierten validierten Instrumente zur Pflegequalitätsmessung, die Betreuung in ihren Beschreibungen mit einschließen, evaluieren zum einen organisations- und personalbezogene Faktoren [27, 28, 29] oder fokussieren sich auf eine bestimmte Erkrankung wie beispielsweise Demenz [5, 30].

Generell haben diese Instrumente den Anspruch, Outcomes durch gesundheitsbezogene Indikatoren $\mathrm{zu}$ messen [20] oder beziehen sich beispielsweise auf die Verhütung von Gesundheitsproblemen wie Stürze oder Schmerzen [31].

Z Gerontol Geriat 2013 · 46:532-542 DOI 10.1007/s00391-012-0463-7

(c) Springer-Verlag Berlin Heidelberg 2013

\section{A. Koppitz $\cdot$ J. Dreizler $\cdot$ H. Hediger $\cdot$ J. Voss $\cdot$ L. Imhof \\ Betreuungsindex in Pflegeheimen. \\ Entwicklung und Validierung eines neuen Instruments zur Beurteilung von Betreuungsqualität in Pflegeheimen}

\section{Zusammenfassung}

Hintergrund. Derzeit gibt es in der Literatur kein geeignetes Instrument zur Beurteilung der Betreuungsqualität in Pflegeheimen. Die Autoren haben deshalb ein neues Instrument, den Betreuungsindex in Pflegeheimen (Nursing Home Care Index, $\mathrm{NCl}$ ), konzipiert und getestet.

Material und Methode. Betreuungsqualität wird in der Literatur in 8 Dimensionen definiert. Es wurde ein Fragebogen mit 42 Fragen aus 12 validierten Messinstrumenten entwickelt. Der Originalfragebogen wurde mit 320 Mitarbeitern aus 15 Alteneinrichtungen getestet. Die Daten wurden mithilfe einer Faktorenanalyse und Cronbachs a untersucht und auf 3 Faktoren und 16 Fragen reduziert. Das revidierte Instrument wurde in einer weiteren Studie mit 136 Mitarbeitern auf seine Anwendbarkeit überprüft.

Ergebnisse. Als Ergebnis der Faktorenanalyse konnten 16 Items mit einer 3-FaktorenStruktur, d. h. soziale Teilhabe, emotionales Wohlbefinden und Selbstbestimmung, identifiziert werden. Diese 3 Faktoren erklären $51,2 \%$ der Gesamtvarianz. Die Reliabilität der Gesamtskala beträgt 0,87, die der 3 Subskalen Selbstbestimmung 0,86 , emotionales Wohlbefinden 0,71 und soziale Beziehungen bzw. Teilhabe 0,78. Die Gesamtpunktezahl des $\mathrm{NCl}$ erlaubt eine Kategorisierung der Betreuungsqualität in den 3 Abstufungen gut, ausreichend und dringender Verbesserungsbedarf.

Schlussfolgerungen. Der $\mathrm{NCl}$ hat eine doppelte Funktion. Einerseits dient er Mitarbeitern und dem Heimmanagement als internes Qualitätssicherungsinstrument. Andererseits könnte es zukünftig Angehörigen von potenziellen Heimbewohnern die Möglichkeit bieten, die Betreuungsqualität verschiedener Pflegeheime zu vergleichen.

Schlüsselwörter

Sicherung der Versorgungsqualität · Pflege · Alte Menschen · Validierungsstudie - Geriatrie

\section{Nursing Home Care Index. Development and validation of a new instrument to evaluate care quality in nursing homes}

\section{Abstract}

Background. There is currently no adequate tool in the literature for assessing the quality of care in nursing homes. Therefore, we developed and tested a new instrument the Nursing Home Care Index ( $\mathrm{NCl}$ ).

Methods. Quality of care is defined in the literature by 8 dimensions. An instrument with 42 questions of 12 validated scales was implemented. The new instrument was tested on 320 staff members in 15 nursing homes. The data were examined with the help of factor analysis and Cronbach's a, which reduced the factors to 3 and the questions to 16 . Finally the revised scale was tested in a further pilot study with 136 staff members.

Results. The revised scale consists of

16 items. Based on the factor analysis, a 3-factor structure, namely social relationships, personal well-being, and self-determination were identified. These 3 factors explained $51.2 \%$ of total variance. Overall Cronbach's a was 0.87 . The a reliability for the subscales was 0.86 (self-determination), 71 personal well-being, and 0.78 social relationship, respectively. Based on the $\mathrm{NCl}$ score, quality of care can be categorized into 3 classes: good, adequate, and urgent need for action.

Conclusions. The $\mathrm{NCl}$ has a double function. Nursing staff and management can now use the $\mathrm{NCl}$ to conduct internal quality assurance regarding their caring efforts. In the future, the $\mathrm{NCl}$ can become a useful tool for families and residents to compare the quality of care in different nursing homes.

\section{Keywords}

Health care quality assurance $\cdot$ Nursing care . Aged · Validation studies as topic · Geriatrics 


\begin{tabular}{|c|c|c|c|}
\hline Konzept & Skala & Verwendete Items & Cronbachs a \\
\hline \multirow[t]{2}{*}{ Soziale Einbindung } & Friendship Scale [47] & $\begin{array}{l}6 \text { (Kontaktaufnahme, Isolation, Kontaktmöglichkeiten, } \\
\text { Kontaktintensität, Freundschaften pflegen, Einsamkeit) }\end{array}$ & 0,81 \\
\hline & ENRICHD Social Support Scale [48] & $\begin{array}{l}3 \text { (Kontaktzuverlässigkeit, Empathie und Wertschätzung, } \\
\text { Befriedigung Kontaktbedürfnisse) }\end{array}$ & 0,86 \\
\hline $\begin{array}{l}\text { Gewohnheiten } \\
\text { berücksichtigen }\end{array}$ & Client Satisfaction Questionaire [49] & $\begin{array}{l}2 \text { (Zufriedenheit mit der Dienstleistungen, Zufriedenheit mit } \\
\text { der Qualität der Dienstleistung) }\end{array}$ & $0,83-0,93$ \\
\hline \multirow[t]{4}{*}{ Empowerment } & $\begin{array}{l}\text { ANP-Intervention Questionnaire, ANP-IQ- } \\
\text { ZHAW (unpublished ZHAW, 2010) }\end{array}$ & $\begin{array}{l}4 \text { (Erreichbarkeit der Pflegenden, Partizipation, Zielverwirkli- } \\
\text { chung, Empowerment) }\end{array}$ & $\begin{array}{l}\text { Keine An- } \\
\text { gaben }\end{array}$ \\
\hline & $\begin{array}{l}\text { Allgemeine Selbstwirksamkeitserwartung, } \\
\text { SWE [50] }\end{array}$ & 3 (Widerstandsfähigkeit, Zielerreichung, Selbstvertrauen) & $\begin{array}{l}0,8-0,9 \text { dtsch. } \\
\text { Versionen }\end{array}$ \\
\hline & $\begin{array}{l}\text { WHO Quality of Life - Old and German Version, } \\
\text { WHOQOL-OLD [51] }\end{array}$ & $\begin{array}{l}3 \text { (Eigenverantwortung, Einflussnahme auf allgemeine } \\
\text { Entscheidungen, Einflussnahme auf den eigenen Tod) }\end{array}$ & $0,63-0,89$ \\
\hline & Diabetes Management Self-Efficacy Scale [52] & 1 (Selbstvertrauen) & 0,81 \\
\hline \multirow{3}{*}{$\begin{array}{l}\text { Akzeptanz und } \\
\text { Wertschätzung }\end{array}$} & Family Life Evaluation, SEF [53] & 1 (Akzeptanz) & $0,72-0,92$ \\
\hline & $\begin{array}{l}\text { ANP-Intervention-Questionnaire ANP-IQ- } \\
\text { ZHAW (unpublished ZHAW, 2010) }\end{array}$ & 2 (gegenseitiges Vertrauen, Privatsphäre) & $\begin{array}{l}\text { Keine An- } \\
\text { gaben }\end{array}$ \\
\hline & ENRICHD Social Support Scale [48] & 2 (Kontaktqualität, Empathie) & 0,86 \\
\hline \multirow[t]{3}{*}{ Personenzentrierung } & $\begin{array}{l}\text { Allgemeine Selbstwirksamkeitserwartung, } \\
\text { SWE [50] }\end{array}$ & 1 (Selbstvertrauen) & $\begin{array}{l}0,8-0,9 \text { in den } \\
\text { dt. Versionen }\end{array}$ \\
\hline & Caregiving Burden Scale [54] & $\begin{array}{l}7 \text { (Verfügbarkeit, Belastung, Aktivitätsübernahme, Schwierig- } \\
\text { keiten meistern, Hilfe organisieren, Hilfe koordinieren, Stellver- } \\
\text { tretende Einflussnahme) }\end{array}$ & 0,87 \\
\hline & Family Life Evaluation, SEF [53] & 1 (Verfügbarkeit von Kontakten) & $0,72-0,92$ \\
\hline \multirow{6}{*}{$\begin{array}{l}\text { Organisationsfakto- } \\
\text { ren (Kommunikation } \\
\text { im Team, mit Bewoh- } \\
\text { nern, Zusammen- } \\
\text { arbeit im Alltag) }\end{array}$} & Team Climate Inventory, TKI [55] & $\begin{array}{l}4 \text { (Entlastungsmöglichkeiten, gegenseitige Achtsamkeit, } \\
\text { Veränderungsbereitschaft, Informationsweitergabe) }\end{array}$ & $0,61-0,89$ \\
\hline & Family Life Evaluation, SEF [53] & $\begin{array}{l}9 \text { (Problemlösungsakzeptanz, Problemfindungsprozess; } \\
\text { Problemidentifikation, emotionale Problemlösungsfähigkei- } \\
\text { ten, Verlässlichkeit, Kontrolle, Aufgabenverteilung, Qualität der } \\
\text { Beziehung, Arbeitsatmosphäre, Standardisierung vs. Indivi- } \\
\text { dualisierung, Offenheit und Konstruktivität) }\end{array}$ & $0,72-0,92$ \\
\hline & $\begin{array}{l}\text { Allgemeine Selbstwirksamkeitserwartung, } \\
\text { SWE [50] }\end{array}$ & 1 (Selbstvertrauen) & $\begin{array}{l}0,8-0,9 \text { in den } \\
\text { dt. Versionen }\end{array}$ \\
\hline & $\begin{array}{l}\text { ANP-Intervention-Questionnaire ANP-IQ- } \\
\text { ZHAW (unpublished ZHAW, 2010) }\end{array}$ & 1 (Kontaktaufnahme) & $\begin{array}{l}\text { Keine An- } \\
\text { gaben }\end{array}$ \\
\hline & $\begin{array}{l}\text { WHO Quality of Life - Old and German Version, } \\
\text { WHOQOL-OLD [51] }\end{array}$ & 1 (Zuversicht) & $0,63-0,89$ \\
\hline & Family Care Inventory, FCI [56] & 1 (emotionale Kompetenz) & $0,71-0,91$ \\
\hline
\end{tabular}

Auch bei den identifizierten Instrumenten, die sich explizit auf „caring“ als Konzeptionsgrundlage beziehen, schließen nur drei Skalen pflegebedürftige Personen in Pflegeheimen ein.

Alle identifizierten Instrumente beziehen sich nach der Outcome-Kategorisierung von Doran [32] überwiegend auf funktionale Gesichtspunkte, Sicherheitsaspekte und/oder klinischen Ergebnisse.

Wenn sie sich auf Ergebnisse im Bereich der subjektiven Wahrnehmung („perceptual“) beziehen, dann wird entweder ihre Aussagekraft hinsichtlich Betreuung seitens der Autoren durch einen zu kleinen Stichprobenumfang oder durch ihren enormen Zeitaufwand eingeschränkt [20, 33, 34, 35, 36].

Deshalb wurde ein neues Instrument zur Messung der Betreuungsqualität für pflegebedürftige Personen in Pflegeheimen entwickelt.

\section{Entwicklung und Vorstellung des $\mathrm{NCl}$}

Ausgangspunkt für die Entwicklung des neuen Instruments war der Anspruch ein kurzes und im Heimalltag einfach einsetzbares Messinstrument zu entwickeln. Die theoretische Konzeption bestand aus den acht literaturgestützt entwickelten Betreu- ungsdimensionen. Diese Dimensionen wurden durch Fragen aus 12 validierten Instrumenten zur Lebensqualität operationalisiert (• Tab. 1).

Jede aus den 12 Instrumenten übernommene Frage wurde in einer Fokusgruppe ( $n=3$, Pflege und Betriebswirtschaft) auf ihre Übereinstimmung mit mindestens einer der acht identifizierten Betreuungsdimensionen überprüft.

Die Vor- und Nachbereitung der Fokusgruppe wurde durch ein Projektteam vorgenommen ( $\mathrm{n}=10$, Heimmanagement, Küche, Hauswirtschaft, Administration, Pflege, Hausdienst). Wurde nicht bei mindestens einer Betreuungsdimension eine 


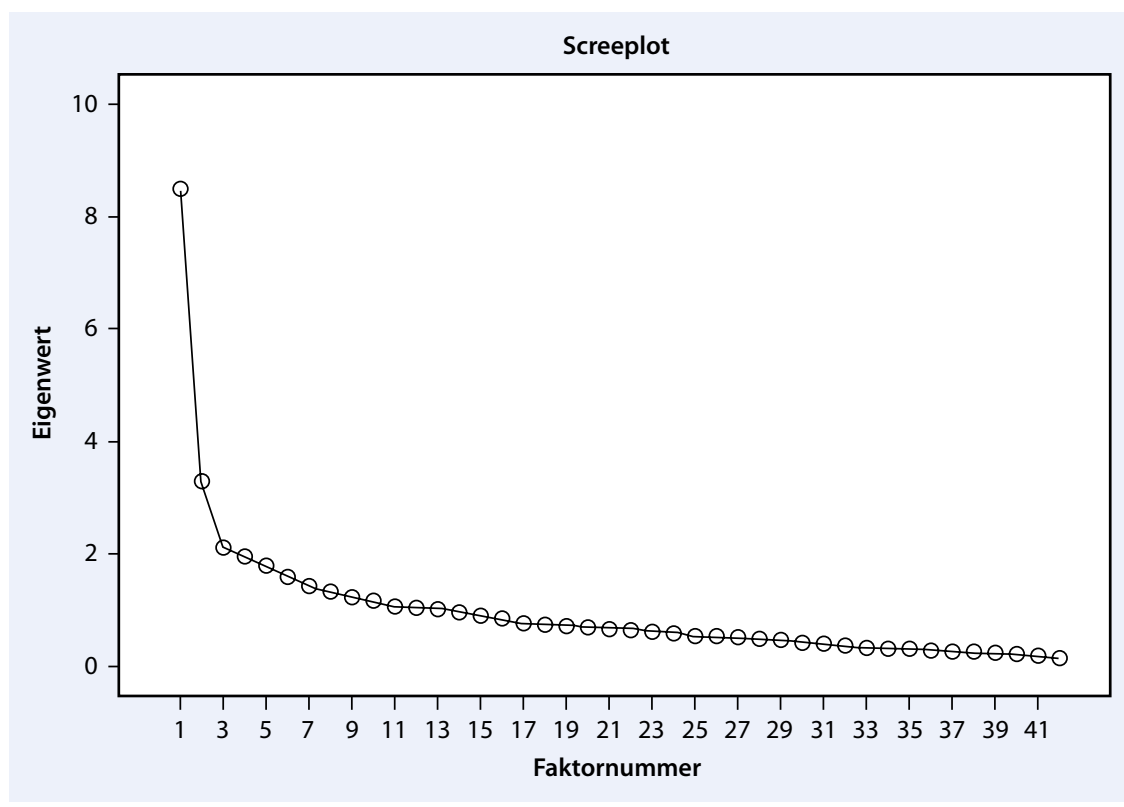

Abb. $3 \Delta$ Screeplot für die 42-Itemversion

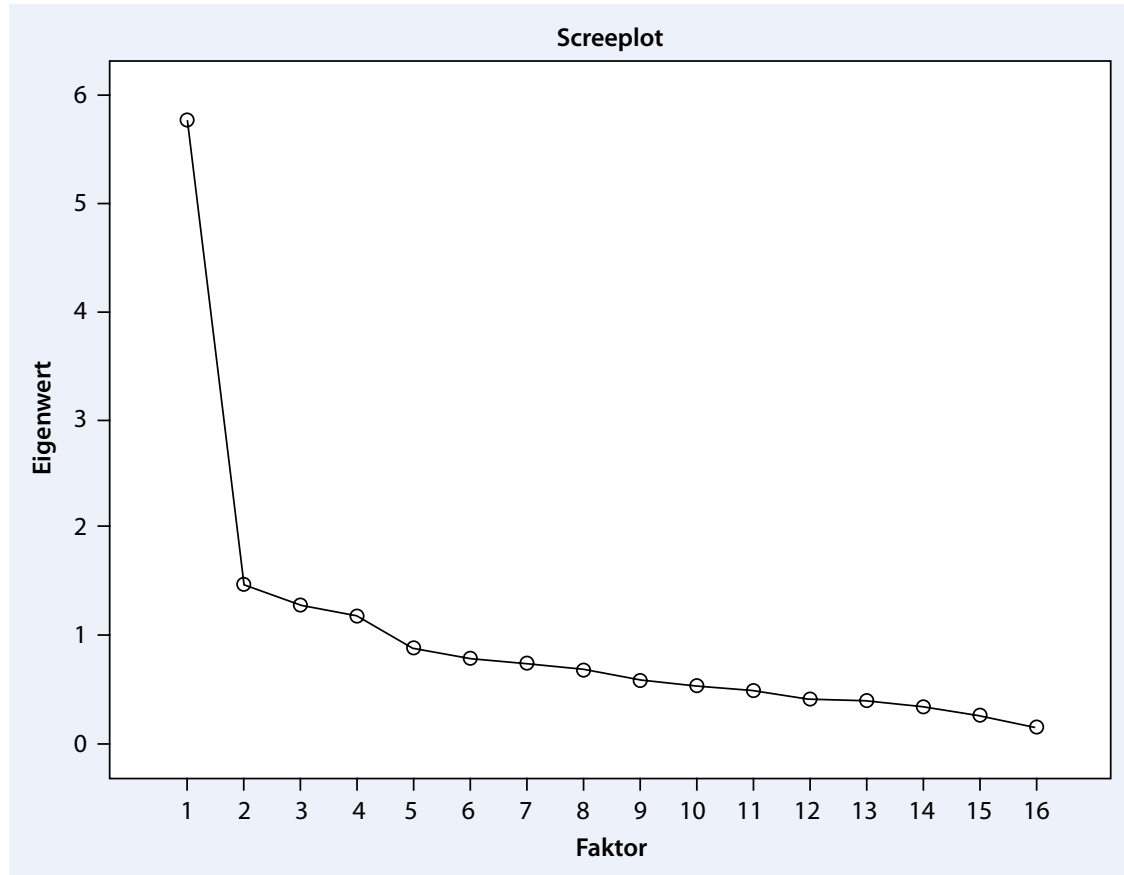

Abb. $4 \Delta$ Screeplot für die 16-Itemversion

Übereinstimmung erkannt, wurde die Frage verworfen.

Nachdem die Fragen für das neu zu entwickelnde Instrument ausgewählt waren, sind diese an die Begrifflichkeiten der stationären Langzeitversorgung pflegebedürftiger Personen angepasst worden. Für jede Frage wurde ein Antwortformat zwischen „0 Punkten“ (trifft überhaupt nicht $\mathrm{zu}$ ) und „100 Punkten“ (trifft voll zu) ge- wählt, um die Betreuungsqualität numerisch mit der addierten Punktzahl aus allen 42 Items erfassen zu können.

Aufbauend auf die Neuentwicklung des Fragebogens waren die Ziele der Studie

- erstens die Überprüfung des Pilotfragebogens „Betreuungsindex in Pflegeheimen“ (Nursing Home Care Index, NCI) auf seine Güte und zweitens die Validierung des gleichnamigen, endgültigen Fragebogens in der Folgestudie, die nachfolgend als Studie 2 bezeichnet wird.

\section{Methode}

Die Sekundärdatenanalyse der quantitativen Querschnittstudie wurde durch eine Zusammenarbeit zwischen der Züricher Hochschule für Angewandte Wissenschaften, Forschung \& Entwicklung Pflege, und dem Verein HeimBenchmarkingSchweiz (HeBeS, http://www.hebes. ch) ermöglicht.

\section{Setting}

In den Jahren 2010 und 2011 beschäftigten sich insgesamt 26 interessierte Pflegeheime aus der Deutschschweiz mit der Thematik Betreuung. Die teilnehmenden Pflegeheime sind für 40 bis 300 Bewohner verantwortlich. Am häufigsten waren Pflegeheime mit einer Größe von 80 bis 120 Bewohnern vertreten.

\section{Datensammlung}

$\mathrm{HeBeS}$ greift mit der Methode des Benchlearning Themen auf, die noch wenig erforscht sind und von der Heim-Praxis als relevant eingeschätzt werden [37]. Interessierte Pflegeheime aus der Deutschschweiz können sich jährlich für eine Teilnahme am Benchlearning entscheiden. Die Zürcher Hochschule (ZHAW) erhielt für eine Sekundäranalyse aus den $\mathrm{He}$ BeS-Benchlearningprojekten in den Jahren 2010 und 2011 die Daten. Für die Piloterhebung im Jahr 2010 wurden 320 Mitarbeiter aus Pflegeeinrichtungen in der Deutschschweiz durch HeBeS zur Studienteilnahme eingeladen. Für die Studie 2 im Jahr 2011 waren es 136. Ein Rückschluss auf die Teilnehmer war für die ZHAW nicht möglich. Eine Genehmigung der zuständigen kantonalen Ethikkommissionen war deshalb nicht notwendig.

\section{Stichprobe}

In die Erhebungen in den Jahren 2010 und 2011 wurden Mitarbeiter aus 15 (2010) und 11 (2011) Pflegeeinrichtungen eingeschlos- 


\begin{tabular}{|c|c|c|}
\hline \multirow[t]{2}{*}{ Merkmal } & \multicolumn{2}{|c|}{ Teilnehmer } \\
\hline & $\begin{array}{l}\text { Anzahl } \\
\text { (n) }\end{array}$ & (\%) \\
\hline \multicolumn{3}{|l|}{ Geschlecht } \\
\hline Weiblich & 120 & 88,2 \\
\hline Männlich & 16 & 11,8 \\
\hline \multicolumn{3}{|l|}{ Alterskategorien in Jahren } \\
\hline$\leq 20$ & 8 & 5,9 \\
\hline $21-30$ & 29 & 21,3 \\
\hline $31-40$ & 23 & 16,9 \\
\hline $41-50$ & 25 & 18,4 \\
\hline $51-60$ & 36 & 26,5 \\
\hline$\geq 61$ & 11 & 8,1 \\
\hline \multicolumn{3}{|l|}{ Bereich/Funktion } \\
\hline Empfang & 1 & 0,7 \\
\hline Küche und Service & 1 & 0,7 \\
\hline Hausdienst & 1 & 0,7 \\
\hline Therapieberufe & 3 & 2,2 \\
\hline Pflegefachpersonen & 27 & 19,9 \\
\hline $\begin{array}{l}\text { Fachangestellte Gesund- } \\
\text { heit/Betreuung }\end{array}$ & 40 & 29,4 \\
\hline Pflegehilfskräfte & 58 & 42,6 \\
\hline \multicolumn{3}{|l|}{ Berufserfahrung } \\
\hline 1-4 Jahre & 33 & 24,3 \\
\hline 5-9 Jahre & 27 & 19,9 \\
\hline$>9$ Jahre & 71 & 52,2 \\
\hline
\end{tabular}

sen, die mindestens 12 Monate bei diesem Träger gearbeitet hatten, mindestens 18 Jahre alt waren und im täglichen Kontakt mit den Bewohnern standen.

Neben der Berufsgruppe der Pflege mit und ohne Pflegediplom wurden Mitarbeiter aus den Bereichen Administration, Küche und Service, Wäscherei und Reinigung und Hausdienst eingeschlossen. Mitarbeiter mit Führungsverantwortung ab der Ebene Bereichsleitung wurden ausgeschlossen.

\section{Datenanalyse}

Die Analysen wurden mittels PASW Statistics Version 19.0 in drei Phasen durchgeführt. Nach der Entwicklung, Test und Diskussion des Pilotinstruments mit Experten aus dem Heimbereich im Jahr 2010 (Phase 1) wurde eine Faktorenanalyse (Phase 2) durchgeführt. In Phase 3 wurde der Fragebogen erneut auf seine Inhaltsund Konstruktvalidität getestet.

\section{Phase 1}

Zur Prüfung der Inhaltsvalidität wurden in Einzelinterviews mit Experten aus dem Heimbereich $(n=4$, Pflegefachperson, Pflegedienst- und Heimleitung, Geschäftsführung) Inhalt, Anwendbarkeit und Praktikabilität des Fragebogens im Alltag diskutiert [38].

\section{Phase 2}

Nach Abschluss der ersten Datensammlung 2010 wurde eine Faktorenanalyse mit einer Hauptkomponentenanalyse und Varimaxrotation in mehreren Schritten durchgeführt. Vor Überprüfung der Konstruktvalidität mittels einer Faktorenanalyse wurde eine mögliche Korrelation zwischen den Items mit dem Bartlett-Test ermittelt. Die gemeinsame Streuung von Items wurde durch den Kaiser-Meyer-Olkin-Test überprüft.

Basierend auf den Mittelwerten und Standardabweichungen wurden zunächst 10 Fragen eliminiert. Aufgrund von Signifikanzwerten und Korrelationen $<0,30$ wurden weitere 7 Fragen isoliert. Nochmals 9 Fragen wurden aufgrund von Cronbachs- $\alpha$-Werten $<0,70$ im Rahmen von Reliabilitätsüberprüfungen extrahiert. Der Screeplot für die 42-Itemversion kann der - Abb. 3 entnommen werden.

\section{Phase 3}

Der 16-Itemfragebogen wurde mit Experten aus dem Heimbereich ( $\mathrm{n}=2$, Pflegefachperson und Pflegedienstleitung) auf Inhaltsvalidität geprüft und nochmals in der Praxis erprobt [38]. Ebenso wurde erneut die Konstruktvalidität durch eine Faktorenanalyse mit einer Hauptkomponentenanalyse und Varimaxrotation durchgeführt. Die Reliabilität wurde auch überprüft. Der dazugehörende Screeplot findet sich in $\bullet$ Abb. 4.

Auf eine Test-Retest-Reliabilität musste im Rahmen dieser Sekundäranalyse verzichtet werden, da die Daten aus zwei Benchlearningprojekten zur Verfügung gestellt wurden und in diesem Kontext keine Wiederholung der Befragung in einem Zeitraum zwischen zwei und sechs Wochen möglich war.

\section{Ergebnisse}

\section{Demografische Daten}

Die Rücklaufquote bei der Befragung 2010 betrug $81 \%(n=259)$. Bei der Folgeerhebung im Jahr 2011 lag die Rücklaufquote bei $100 \%(n=136)$. Mehr als die Hälfte (52\%) der Teilnehmer waren bei der Befragung 201141 Jahre und älter. Fast Zweidrittel aller Mitarbeiter (72\%) hatten eine mehr als fünfjährige Berufserfahrung. Weiblich waren $88 \%$. Die $\bullet$ Tab. 2 gibt einen Überblick der Teilnehmer der Studie 2 aus dem Jahr 2011.

\section{Itemreduktion}

Bereits der Pilotfragebogen aus dem Jahr 2010 wies gute Cronbachs- $\alpha$-Werte für das Gesamtinstrument von 0,86 aus. Die beiden Ergebnisse aus dem Bartlett- und Kaiser-Meyer-Olkin-Test erlaubten, die Faktorenanalyse mit Hauptkomponentenextraktion- und Varimaxrotationsmethode des Pilotfragebogens mit 42 Items durchzuführen.

Diese Analyse ergab eine Reduzierung des Fragebogens auf 16 Fragen mit 3 Subskalen (Betreuungsdimensionen). Der endgültige Fragebogen mit den 16 Fragen und einer Antwortkategorie von „0 Punkten“ (trifft überhaupt nicht zu) bis „,100 Punkten“ (trifft voll zu) kann der - Tab. 3 entnommen werden.

\section{Drei-Faktoren Struktur}

Als Endresultat konnte eine stabile DreiFaktoren-Struktur mit 16 Items gewonnen werden, die sich auch bei der erneuten Faktorenanalyse mit den Daten aus 2011 bestätigt hat. Diese drei Faktoren bzw. Subskalen wiesen Eigenwerte $>1$ auf. Sie erklärten gemeinsam $51,2 \%$ der Varianz im Gesamtmodell bzw. 53,3\% mit den Daten aus 2011. Faktor 1 erklärte 23,3\% der Varianz (2011: 20,6\%), Faktor 2 16,3\% (2011: 18,3\%) und Faktor 3 11,6\% (2011: 14,4\%) im Gesamtmodell.

$\mathrm{Zu}$ einer Subskala wurden die Items zugewiesen, die mindestens mit einem Wert von 0,45 auf je nur einen Faktor geladen haben und deshalb als Markierungsitems bezeichnet werden konnten [39]. ๑ Tab. 4 können die entsprechen- 
Tab. 3 Betreuungsindex (Nursing Home Index, NCI). Alle Aussagen verfügen über dieselben Zustimmungsmöglichkeiten: trifft überhaupt nicht zu, trifft voll zu

1. Viele Bewohner haben bei uns Freunde gefunden.

2. Jeder Bewohner hat eine Gruppe, zu der er sich zugehörig fühlt.

3. Jeder Bewohner hat eine Person, um Intimes zu besprechen.

4. Wenn ein Bewohner verstimmt ist, wissen wir warum.

5. Jeder Bewohner hat so viele Kontaktmöglichkeiten, wie sie sich dies wünscht.

6. Die Bewohner fühlen sich bei uns wie Zuhause.

7. Die Bewohner dürfen bei uns ihr Eigensein („,Mödeli") beibehalten.

8. Unser Tagesablauf gibt den Bewohnern Sicherheit.

9. Die Bewohner können unseren Tagesablauf mitbestimmen.

10. Unsere Bewohner werden unterstützt, ihre Probleme aus eigener Kraft zu lösen.

11. Unsere Bewohner können ihre eigenen Entscheidungen treffen.

12. Unsere Bewohner können die Dinge tun, die sie gern tun.

13. Unsere Bewohner können ihre eigenen Ziele verfolgen.

14. Wir teilen mit unseren Bewohnern Freuden und Leiden.

15. Wenn sich unsere Bewohner bedrückt fühlen, wissen sie, an wen sie sich wenden können.

16. Ich kann auf unverhofft geäußerte Wünsche unserer Bewohner jederzeit eingehen.

den Kennwerte der Daten aus 2010 entnommen werden.

Die Faktorstabilität betrug 0,89 , was Bortz [40] als gute Faktorenstruktur bezeichnet. Er empfiehlt, dass Faktorenstrukturen mit $<0,8$ nicht interpretiert werden sollten bzw. eine gute Übereinstimmung zwischen tatsächlicher und stichprobenbedingter Faktorenstruktur bei $\geq 0,9$ liegt. Dies wird in der vorliegenden Faktorenstruktur erreicht.

Jede Subskala wurde auf seine interne Konsistenz durch Cronbachs a geprüft. Die Subskala "Selbstbestimmung“ wies einen Cronbachs- $\alpha$-Wert von 0,86, die Subskala „emotionales Wohlbefinden" den Wert von 0,71 und die Subskala „soziale Teilhabe“ den Wert von 0,78 auf. Der Cronbachs- $\alpha$-Wert des Gesamtbetreuungsindexes lag bei Wert 0,87 . Alle Trennschärfekoeffizienten wiesen akzeptable Werte zwischen 0,3 und 0,8 auf [40].

Es wurden keine altersabhängigen und/oder Unterschiede in der Berufserfahrung festgestellt (• Tab. 5). Bei der Indexbildung wurde die Homogenität der Faktorladung berücksichtigt.

Da von einer Gleichwertigkeit der einzelnen Items ausgegangen wird, wird der NCI-Gesamtindex durch die Addition aller 16 Itemwerte gebildet. Pro Teilnehmer können maximal 1600 Punkte erreicht werden. Für die Berechnung der NCI-Gesamtpunktzahl für eine Einrichtung wird der Gesamtindex NCI durch die Addition aller Itemwerte pro Teilnehmer errechnet und durch die Gesamtzahl der Teilnehmer dividiert. Der NCI-Gesamtindex für eine Einrichtung mit einem Gesamtscore - zwischen 70 und $100 \%$ wird als "gut",

- zwischen 50 bis unter $70 \%$ als ,ausreichend" und

- unterhalb von $50 \%$ als „dringender Verbesserungsbedarf" bezeichnet.

Die eindeutige Zuweisung von Items in jeweils nur eine von drei Subskalen erklärt die Reduzierung von ursprünglich acht auf drei Subskalen.

\section{Subskala 1}

Bei der Subskala 1 (Selbstbestimmung) geht es darum über seine eigenen Lebensbezüge und Gewohnheiten selbst bestimmen zu können und damit um die Sicherheit die Um- und Mitwelt im Heim selbst zu gestalten. Der Schutz vor Einflüssen von außen, über die keine Kontrolle erlangt werden kann, steht im Vordergrund. Die Subskala 1 setzt sich aus den folgenden 8 Items zusammen:

- Kontakte wunschgemäß gestalten können,

- sich zuhause fühlen,

- Gewohnheiten beibehalten können,

- einen Tagesablauf, der Sicherheit bietet,

- Unterstützung, um Probleme aus eigener Kraft zu lösen,

- Entscheidungen, die durch Betroffene gefällt werden bzw. in deren Sinn sind,
- Dingen zu tun, die im Interesse des Betroffenen sind, und

- Ziele, die der Betroffene auch tatsächlich anvisiert.

Die Subskala 1 wird deshalb als „Selbstbestimmung" bezeichnet.

\section{Subskala 2}

Subskala 2 (emotionales Wohlbefinden) beinhaltet 6 Items, die die Bedeutung der Lebenszufriedenheit im gegenwärtigen Leben widerspiegeln.

$\mathrm{Zu}$ den Teilaspekten des emotionalen Wohlbefindens zählen u. a.

- Intimes besprechen zu können und zu wissen, warum z. B. der Gesprächspartner gerade verstimmt ist,

- über den Tagesablauf bestimmen zu können,

- Freuden und Leiden mit jemanden teilen zu können bzw. eine Person als Ansprechpartner bei Kummer zu haben, als auch

- Wünsche zu haben, die erfüllt werden können.

\section{Subskala 3}

Subskala 3 (soziale Teilhabe) schließlich beinhaltet zwei Aspekte,

- die Zugehörigkeit zu einer sozialen Gruppe und

- das eingebunden sein in eine Gruppe.

Freundschaften schließen und pflegen und sich einer Gruppe zugehörig fühlen, wird deshalb als „soziale Teilhabe“ bezeichnet.

\section{Diskussion}

Der Betreuungsindex in Pflegeheimen, NCI, beinhaltet eine stabile Drei-Faktoren-Struktur mit 16 Items zur Messung von Betreuungsqualität. Eine ausreichende erste Evidenz des NCI konnte in zwei Stichproben mit insgesamt 395 Teilnehmern (Studie $1 \mathrm{n}=259$, Studie $2 \mathrm{n}=136$ ) nachgewiesen werden.

Die Konstrukt- und Inhaltsvalidität wurden in beiden Stichproben überprüft. Der Pilotfragebogen aus der Studie $1 \mathrm{mit}$ 42 Items und 8 Dimensionen zur Betreuung konnte in der Studie 2 nicht bestätigt werden. 


\begin{tabular}{|c|c|c|c|c|c|c|c|c|}
\hline \multicolumn{5}{|l|}{ Rotierte Faktorladungen } & \multicolumn{4}{|c|}{ Itemkennwerte } \\
\hline \multirow[t]{2}{*}{ Item } & \multirow[t]{2}{*}{ Frage } & \multicolumn{3}{|l|}{ Subskala } & \multirow[t]{2}{*}{$\begin{array}{l}\text { Schwie- } \\
\text { rigkeit }\end{array}$} & \multicolumn{3}{|c|}{$\begin{array}{l}\text { Subskalen- } \\
\text { Trennschärfe }\end{array}$} \\
\hline & & $\begin{array}{l}1 \text { Selbstbe- } \\
\text { stimmung }\end{array}$ & $\begin{array}{l}2 \text { Emotionales } \\
\text { Wohlbefinden }\end{array}$ & $\begin{array}{l}3 \text { Soziale } \\
\text { Teilhabe }\end{array}$ & & 1 & 2 & 3 \\
\hline $\begin{array}{l}\text { Q5 } \\
\text { Kontakt }\end{array}$ & $\begin{array}{l}\text { Jeder Bewohner hat so viele Kontaktmög- } \\
\text { lichkeiten, wie er sich dies wünscht. }\end{array}$ & 0,469 & 0,358 & 0,281 & 0,7 & 0,42 & & \\
\hline $\begin{array}{l}\text { Q6 } \\
\text { Zuhause }\end{array}$ & $\begin{array}{l}\text { Die Bewohner fühlen sich bei uns wie Zu- } \\
\text { hause. }\end{array}$ & 0,565 & 0,282 & 0,346 & 0,69 & 0,62 & & \\
\hline $\begin{array}{l}\text { Q7 } \\
\text { Gewohnheiten }\end{array}$ & $\begin{array}{l}\text { Die Bewohner dürfen bei uns ihr Eigensein } \\
\text { („,Mödeli“) beibehalten. }\end{array}$ & 0,643 & 0,135 & 0,299 & 0,79 & 0,64 & & \\
\hline $\begin{array}{l}\text { Q8 } \\
\text { Tagesablauf }\end{array}$ & $\begin{array}{l}\text { Unser Tagesablauf gibt den Bewohnern } \\
\text { Sicherheit. }\end{array}$ & 0,713 & 0,070 & 0,029 & 0,82 & 0,49 & & \\
\hline $\begin{array}{l}\text { Q11 } \\
\text { Problemlösung }\end{array}$ & $\begin{array}{l}\text { Unsere Bewohner werden unterstützt, ihre } \\
\text { Probleme aus eigener Kraft zu lösen. }\end{array}$ & 0,580 & 0,317 & 0,146 & 0,64 & 0,56 & & \\
\hline $\begin{array}{l}\text { Q12 } \\
\text { Entscheidungen }\end{array}$ & $\begin{array}{l}\text { Unsere Bewohner können ihre eigenen } \\
\text { Entscheidungen treffen. }\end{array}$ & 0,657 & 0,161 & 0,017 & 0,72 & 0,60 & & \\
\hline $\begin{array}{l}\text { Q14 } \\
\text { Aktivitäten }\end{array}$ & $\begin{array}{l}\text { Unsere Bewohner können die Dinge tun, } \\
\text { die sie gern tun. }\end{array}$ & 0,696 & 0,247 & $-0,072$ & 0,78 & 0,74 & & \\
\hline $\begin{array}{l}\text { Q15 } \\
\text { Selbstverwirklichung }\end{array}$ & $\begin{array}{l}\text { Unsere Bewohner können ihre eigenen } \\
\text { Ziele verfolgen. }\end{array}$ & 0,704 & 0,276 & 0,107 & 0,66 & 0,76 & & \\
\hline $\begin{array}{l}\text { Q3 } \\
\text { Privates }\end{array}$ & $\begin{array}{l}\text { Jeder Bewohner hat eine Person, um Inti- } \\
\text { mes zu besprechen. }\end{array}$ & 0,164 & 0,563 & 0,449 & 0,55 & & 0,50 & \\
\hline $\begin{array}{l}\text { Q4 } \\
\text { Stimmung }\end{array}$ & $\begin{array}{l}\text { Wenn ein Bewohner verstimmt ist, wissen } \\
\text { wir warum. }\end{array}$ & 0,199 & 0,704 & 0,140 & 0,63 & & 0,54 & \\
\hline $\begin{array}{l}\text { Q10 } \\
\text { Partizipation }\end{array}$ & $\begin{array}{l}\text { Die Bewohner können unseren Tagesab- } \\
\text { lauf mitbestimmen }\end{array}$ & 0,357 & 0,456 & 0,211 & 0,58 & & 0,34 & \\
\hline $\begin{array}{l}\text { Q21 } \\
\text { Freud und Leid }\end{array}$ & $\begin{array}{l}\text { Wir teilen mit unseren Bewohnern Freuden } \\
\text { und Leiden. }\end{array}$ & 0,090 & 0,689 & $-0,089$ & 0,83 & & 0,31 & \\
\hline $\begin{array}{l}\text { Q22 } \\
\text { Bezugsperson }\end{array}$ & $\begin{array}{l}\text { Wenn sich unsere Bewohner bedrückt } \\
\text { fühlen, wissen sie, an wen sie sich wenden } \\
\text { können. }\end{array}$ & 0,347 & 0,589 & 0,067 & 0,78 & & 0,54 & \\
\hline $\begin{array}{l}\text { Q23 } \\
\text { Zufriedenheit }\end{array}$ & $\begin{array}{l}\text { Ich kann auf unverhofft geäußerte } \\
\text { Wünsche unserer Bewohner jederzeit ein- } \\
\text { gehen. }\end{array}$ & 0,411 & 0,475 & 0,047 & 0,74 & & 0,48 & \\
\hline $\begin{array}{l}\text { Q1 } \\
\text { Freunde }\end{array}$ & $\begin{array}{l}\text { Viele Bewohner haben bei uns Freunde } \\
\text { gefunden. }\end{array}$ & 0,148 & $-0,076$ & 0,773 & 0,57 & & & 0,64 \\
\hline $\begin{array}{l}\text { Q2 } \\
\text { Zugehörigkeit }\end{array}$ & $\begin{array}{l}\text { Jeder Bewohner hat eine Gruppe, zu der er } \\
\text { sich zugehörig fühlt. }\end{array}$ & 0,009 & 0,199 & 0,805 & 0,54 & & & 0,64 \\
\hline Cronbachs-a-Subskalen & & 0,86 & 0,71 & 0,78 & & & & \\
\hline Cronbachs-a-Gesamtindex & & 0,87 & & & & & & \\
\hline
\end{tabular}

Die theoretische Konzeption zur Betreuung wurde im Rahmen der Konstruktvalidierung auf 3 Dimensionen reduziert

- Selbstbestimmung,

- emotionales Wohlbefinden und

- soziale Teilhabe.

Fromm [39] empfiehlt, Faktoren mit Ladungen unter 0,45 auszuschließen. Zudem sollte jedes Item aus den jeweiligen Subskalen nur einmal vorkommen, um tatsächlich als Markierungsindex zu fungieren [39].
Die Subskala 3, soziale Teilhabe, enthält nur 2 Items, diese jedoch mit einem Cronbachs- $\alpha$-Wert von 0,78 , was von mehreren Autoren übereinstimmend als gut bezeichnet wird [39, 40, 41].

Diese drei Betreuungsdimensionen finden sich zudem alle in den zum Konzept „care“ und „caring“ identifizierten Messinstrumenten wieder [33, 34, 35, 36, 42]. Auch in den Instrumenten von Wingenfeld u. Engels [20] und Wahl u. Oswald [43] sind die drei Betreuungsdimensionen enthalten und bestätigen unsere Konzeption von Betreuungsqualität. Außerdem entsprechen die Dimensionen soziale Teilhabe, emotionales Wohlbefinden und Selbstbestimmung, als die zentralen Faktoren des Betreuungsindexes, zudem auch dem Konzept der personenzentrierten Pflege nach Kitwood [3] und dem „Caring-Konzept" [21, 22, 44, 45, 46].

Auf eine Anpassung der Definition von Betreuung konnte deshalb auch verzichtet werden, weil in den drei Betreuungsdimensionen weiterhin eine Synthese aus körperorientierten, unterstützenden Handlungen und psychosozialer Unterstützung zum Ausdruck kommt. 
Tab. 5 Vergleich nach Alter und Berufserfahrung

\begin{tabular}{|lllll}
\hline & Alter & \multicolumn{3}{c}{ Beruf } \\
\hline & Teststatistik & Signifikanz $(\mathbf{p})$ & Teststatistik & Signifikanz \\
\hline Subskala 1 & $\mathrm{Z}=1,021$ & 0,307 & $\mathrm{~T}=1,649$ & 0,102 \\
\cline { 2 - 5 } & & & $(\mathrm{df}=116)$ & 0,189 \\
\hline Subskala 2 & $\mathrm{Z}=-0,425$ & 0,671 & $\mathrm{~T}=1,320$ & \\
\cline { 2 - 5 } & & & $(\mathrm{df}=127)$ & 0,206 \\
\hline Subskala 3 & $\mathrm{T}=-0,840$ & 0,402 & $\mathrm{Z}=-1,265$ & \\
\cline { 2 - 5 } & $(\mathrm{df}=128)$ & & & \\
\end{tabular}

Die größte Differenz zu den drei identifizierten "Caring-Instrumenten“ von Marini (Caring Behavior Assessment, [33]), Smith (Caring Assessment Instrument, [42]) und Wolf (Caring Behavior Inventory for Elderly, [36]) liegt in der Anzahl der Items und Subskalen und in der Stichprobengröße.

Das Instrument Caring Behavior Assessment hat 64 Items. Das Caring Behavior Assessment enthält 50 Items mit 6 Subskalen. Außerdem fehlt eine konzeptionelle Zuordnung. Der Caring Behavior Inventory for Elderly enthält 28 Items, wird jedoch in den Anwendung als zeitaufwändig beschrieben. Der Betreuungsindex in Pflegeheimen, NCI, nimmt pro Teilnehmer zwischen 15 bis 20 min in Anspruch.

Der NCI hat zudem den Vorteil, dass es dem von Doran [32] definierten Outcomebereich „perceptual“ (Wahrnehmung) entspricht. Bei den von Benner [46] und Kitwood [3] beschriebenen Interaktionsqualitäten ist dies als besonders relevant zu betrachten. Nicht nur zählbare Handlungen haben einen Einfluss auf die Betreuung, sondern auch der Interaktionsprozess und die Art der Handlungen im Heimalltag. Beide Aspekte kommen in der Haltung der Mitarbeiter zum Ausdruck. Mit dem NCI werden diese Punkte in eine quantifizierbare Maßeinheit transferiert.

Den Autoren ist dabei bewusst ist, dass diese Art der Quantifizierung nie ein vollständiges Abbild der von der Bewohnern erlebten Betreuungswirklichkeit widerspiegeln kann. Ebenso wurde durch die Definition von Betreuung deutlich, dass Betreuung ein Produkt ist, das durch die Interaktion zwischen pflegebedürftiger Person und Mitarbeiter entsteht.

Die 16 Items des NCI gehen ausschließlich auf die Bereiche ein, die von einem Pflegeheim direkt beeinflussbar sind. Für Wingenfeld [20] war dies bei der Entwicklung von Outcomekriterien ebenfalls handlungsleitend.

Für den Einsatz im Regelbetrieb und für Repräsentativität der Aussagen für ein Pflegeheim ist es zudem wichtig, dass alle pflegebedürftigen Personen eingeschlossen werden. Die Daten können mit geringem Arbeitsaufwand in bestehenden Routineabläufen mit einer üblichen Rechenstandardsoftware durch die Einrichtung nicht nur erhoben, sondern auch ausgewertet werden. Dies haben Anwendungstests bei den Benchlearningpartnern ergeben. Dies kann als eine wichtige Voraussetzung für den Einsatz als internes Qualitätssicherungsinstrument bezeichnet werden.

In einem weiteren Entwicklungsschritt könnte der NCI zum Vergleich der Betreuungsqualität unter den Einrichtungen eingesetzt werden. Im Sinne der Verbrauchertransparenz wäre der NCI damit nicht nur für das einzelne Pflegeheim und dessen interne Qualitätssicherung ein Mittel der Wahl, sondern der Verbraucher würde mit dem Index ein verständliches und handhabbares Instrument erhalten.

Grundvoraussetzung hierzu ist eine repräsentative Stichprobe von Pflegeheimen eines Kantons, Bezirks oder Bundeslands. So wäre gewährleistet, dass materielle und immaterielle Ressourcen ähnlich verteilt sind und Einrichtungen mit gleichen Voraussetzungen verglichen werden.

Eine öffentliche Qualitätsberichtserstattung oder externe Qualitätsprüfungen zur Betreuungsqualität, wie sie in Deutschland im Rahmen der Heim- und Pflegegesetzgebung durchgeführt werden, sind in der Schweiz weder Gegenstand in der Gesetzgebung noch wird hier eine öffentlich wahrnehmbare Debatte geführt.

Werden die Ergebnisse des NCI von den Pflegeheimen staatlichen Institutio- nen oder Versicherern unter der genannten Grundvoraussetzung zur Verfügung gestellt, könnten sie in die Qualitätsberichterstattung bzw. -prüfungen aufgenommen werden. In diesem Zusammenhang soll aber nochmals betont werden, dass das primäre Ziel bei der Entwicklung des NCI der Einsatz als internes Qualitätssicherungsinstrument war.

Wenn Pflegeheime selbst dazu übergehen und ihre Ergebnisse nicht nur intern vergleichen, sondern gezielt für ihre Öffentlichkeitsarbeit und für die Werbung von Pflegefachpersonen einsetzen, ist dies für alle Beteiligten umso erfreulicher. Dies konnte im beschriebenen Benchlearningprojekt von $\mathrm{HeBeS}$ beobachtet werden.

\section{Limitationen}

Die Itemstruktur konnte in den zwei vorgestellten Studien bestätigt werden. Offen ist jedoch, ob die Bewertung der Gesamtpunkte des NCI mit "gut", „,ausreichend“ und „dringender Verbesserungsbedarf “ anwendbar ist. Sie muss in einer Folgestudie empirisch getestet werden, da diese Dreiteilung ausschließlich eine theoretische Annahme darstellt.

Offen ist zudem, welche Aussagekraft die isolierten Subskalen haben und wie die Subskalen untereinander bewertet werden.

Der Stichprobenumfang sollte in der Folgestudie mindestens 210 Teilnehmer betragen, da von einer durchschnittlichen Ausfallquote von etwa $30 \%$ ausgegangen werden kann. In den beiden Studien war die Motivation der Teilnehmer durch die freiwillige Teilnahme an einem Benchlearningprojekt sehr hoch, was nicht immer als gegeben vorausgesetzt werden kann.

Die Skalierung der Antworten mit Werten von 0 bis 100 Punkten muss ebenfalls einer kritischen Überprüfung unterzogen werden. Eine Antwortskalierung zwischen 0 und 10 Punkten würde die Vielfalt von Antwortbreitmöglichkeiten weiterhin sichern, wäre aber in der Auswertung einfacher zu handhaben.

Die 16 Items des Betreuungsindexes NCI messen das Konzept Betreuung nicht direkt durch die Einschätzung von Bewohnern. Dies ist eine deutliche Einschränkung, die bei einer Weiterentwick- 
lung des neuen Instruments berücksichtigt werden sollte.

In einem ersten kleinem Pilotversuch $(n=3)$ wurden die pflegebedürftigen Personen durch ein Interview zu den 16 Items des NCI befragt. Dies lässt darauf schließen, dass die Fragen auch für die Betroffenen verständlich sind.

Die konsequente Orientierung an den Bewohnern ist das zentrale Ziel in der Gesundheitsversorgung. Primär sollte die Beurteilung der Betreuungsqualität auch von den Bewohnern beurteilt werden, wenngleich kognitive Einschränkungen hier eine große Hürde darstellen können.

Eine weitere Limitation ist die mit 2 Items repräsentierende Subskala 3. Diese Skala wurde dennoch als relevant betrachtet, da sie einen hohen Cronbachsa-Wert von 0,78 und hohe Faktorladungen (• Tab. 4) aufweist.

Die Frage 7 zu den Gewohnheiten von Bewohnern, wurde mit dem dialektspezifischen Begriff „Mödeli“ angereichert. In der Anwendung in anderen deutschsprachigen Ländern muss diese Begrifflichkeit angepasst werden.

\section{Fazit für die Praxis}

- Soziale Teilhabe, emotionales Wohlbefinden und Selbstbestimmung sind die Eckpfeiler einer guten Betreuungsqualität und werden durch die 16 Items des $\mathrm{NCl}$ operationalisiert. Damit wird Betreuung für Bewohner, Angehörige und Mitarbeiter handhabbar, verständlich und sichtbar.

- Betreuung ist nicht nur einer Berufsgruppe oder einem Bereich, in schweizerischen Pflegeheimen wird von „Aktivierung und Betreuung" gesprochen, zuzuordnen, sondern wird von allen Mitarbeiten geleistet.

- Betreuung ist ein gemeinsames Produkt von Bewohner und Mitarbeitern.

- Der NCl ist ein Instrument zur Outcome-Messung.

- Aus Verbrauchersicht kann der $\mathrm{NCl}$ in Zukunft dazu dienen, die Betreuungsqualität zwischen Pflegeheimen vergleichbar zu machen.

- Das Heimmanagement erhält mit dem $\mathrm{NCl}$ einen Indikator zur internen Qualitätssicherung.

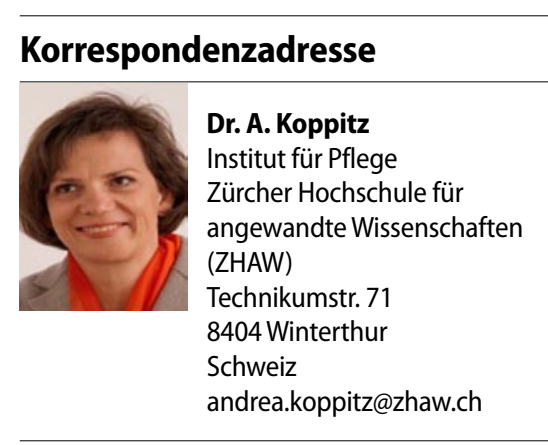

Danksagung. Wir danken der Schiller Stiftung, www.paul-schiller-stiftung.ch, für die finanzielle Unterstützung bei der Piloterhebung im Jahr 2010. Wir danken Ruth Köppel, Geschäftsführung HeBeS, http://www.hebes.ch, für die Möglichkeit der Sekundärdatenanalyse.

Drittmittel: Paul-Schiller-Stiftung

Interessenkonflikt. Die korrespondierende Autorin gibt für sich und ihre Koautoren an, dass kein Interessenkonflikt besteht.

\section{Literatur}

1. Höpflinger F, Bayer-Oglesbay L (2011) Pflegebedürftigkeit und Langzeitpflege im Alter. Buchreihe des Schweizerischen Gesundheitsobservatoriums, Huber, Bern

2. Bayer-Oglesbay L, Höpflinger F (2010) Statistische Grundlagen zur regionalen Pflegeheimplanung in der Schweiz. In: Obsan (Hrsg) Methodik und kantonale Kennzahlen, in Obsan Bericht Nr. 47, S.G., Schweizerisches Gesundheitsobservatorium: Neuchâtel

3. Kitwood TM (2002) Demenz: der person-zentrierte Ansatz im Umgang mit verwirrten Menschen, Huber, Bern [u.a.]

4. Becker S, Kaspar R, Kruse A (2006) Die Bedeutung unterschiedlicher Referenzgruppen für die Beurteilung der Lebensqualität demenzkranker Menschen. Z Gerontol Geriatr 39(5):350-357

5. Becker S, Kaspar R, Kruse A (2011) H.I.L.DE: Heidelberger Instrument zur Erfassung der Lebensqualität demenzkranker Menschen (H.I.L.DE.). 1. Aufl., Huber, Bern 168

6. Kruse A (2005) Selbständigkeit, bewusst angenommene Abhängigkeit, Selbstverantwortung und Mitverantwortung als zentrale Kategorien einer ethischen Betrachtung des Alters. Z Gerontol Geriatr 38(4):273-287

7. Kruse A, Wahl HW (1999) II. Persönlichkeitsentwicklung im Alter. Z Gerontol Geriatr 32(4):279293

8. Kruse A, Wahl HW (1999) III. Soziale Beziehungen. Z Gerontol Geriatr 32(5):333-347

9. Perrig-Chiello P (1997) Wohlbefinden im Alter: körperliche, psychische und soziale Determinanten und Ressourcen, Juventa, Weinheim

10. Zürich K (2010) Pflegegesetz Kantonsrat Zürich, Zürich

11. Maslow AH (2008) Motivation und Persönlichkeit. 11. Aufl., Rowohlt, Reinbek bei Hamburg 395

12. Eichhorn S (1967) Krankenhausbetriebslehre 1, Kohlhammer, Stuttgart

13. Eichhorn S (1975) Krankenhausbetriebslehre: Theorie und Praxis des Krankenhausbetriebes, Kohlhammer, Stuttgart
14. Müller E (2001) Leitbilder in der Pflege: Eine Untersuchung individueller Pflegeauffassungen als Beitrag zu ihrer Präzisierung, Bern, Huber

15. Walker LO, Avant KC (2011) Strategies for theory construction in nursing, Pearson Prentice Hall, Boston (etc)

16. Wilson J (2006) Thinking with concepts, Cambridge (Eng.): University Press

17. Watson J (2009) Assessing and measuring caring in nursing and health sciences. 2. Aufl., ed. I. Watson Caring Science, Springer Pub. Co., New York

18. Schumacher J, Klaiberg A, Brähler E (2003) Diagnostische Verfahren zu Lebensqualität und Wohlbefinden, Göttingen Hogrefe, Verlag für Psychologie

19. Kane RA (2003) Definition, measurement, and correlates of quality of life in nursing homes: toward a reasonable practice, research, and policy agenda. Gerontologist 43(2):28-36

20. Wingenfeld K, Engels S (2011) Entwicklung und Erprobung von Instrumenten zur Beurteilung der Ergebnisqualität in der stationären Altenhilfe, In: B.f.F. Bundesministerium für Gesundheit, Senioren, Frauen und Jugend (Hrsg) Bundesministerium für Gesundheit, Bundesmininsterium für Familien, Senioren, Frauen und Jugend, Berlin

21. Benner P, Wrubel J (1989) The Primacy of Caring: Stress and Coping in Health and IIIness, Menlo Park, Addison-Wesley

22. Benner P, Wrubel J (1997) Pflege, Streß und Bewältigung. Gelebte Erfahrung von Gesundheit und Krankheit, Huber, Bern

23. Fosbinder D (1994) Patient perceptions of nursing care: an emerging theory of interpersonal competence. J Adv Nurs 20(6):1085-1093

24. Schnepp W (2006) Pflegekundige Sorge. Pflege und Gesellschaft 1(2):13-16

25. Hoffmann F et al (2011) Quality management by result-oriented indicators: towards benchmarking in residential care for older people, E.Z.f.W.u. Sozialforschung, Wien

26. Wahl HW (2009) Lebensqualität von Heimbewohnern, in Pflegebedarf und Versorgungssituation bei älteren Menschen in Heimen. In: Wahl HW, Schneekloth U Kohlhammer, Stuttgart. S $30 \mathrm{ff}$

27. Willemse BM et al (2011) Nursing home care for people with dementia and residents' quality of life, quality of care and staff well-being: design of the Living Arrangements for people with Dementia (LAD)-study. BMC Geriatr 11:S 11

28. Rooij AH de et al (2011) Quality of life of residents with dementia in long-term care settings in the Netherlands and Belgium: design of a longitudinal comparative study in traditional nursing homes and small-scale living facilities. BMC Geriatr 11:S 20

29. Castle N, Engberg J (2007) The influence of staffing characteristics on quality of care in nursing homes. Health Res Edu Trust 42(5):1822-1847

30. Dichter M et al (2011) Validity, reliability, and feasibility of a quality of life questionnaire for people with dementia. Z Gerontol Geriatr 44(6):405-410

31. Lin PC et al (2011) Predictors of pain in nursing home residents with dementia: a cross-sectional study. J Clin Nurs 20(13/14):1849-1857

32. Doran D (2011) Nursing outcomes: the state of the science. Jones \& Bartlett Learning, Sudbury

33. Marini B (1999) Institutionalized older adults' perceptions of nurse caring behaviors: a pilot study. J Gerontol Nurs 25(5):10-16

34. Wolf ZR (1986) The caring concept and nurse identified caring behaviors. Topics in Clinical Nursing 8(2):84-93 
35. Wolf ZR et al (2004) Development and testing of the caring behaviors inventory for elders. Int J Hum Care 8(1):48-54

36. Wolf ZR et al (2006) The caring behaviors inventory for elders: development and psychometric characteristics. Int J Hum Care 10(1):49-59

37. Dattakumar R, Jagedeesh R (2003) „A review of literature on benchmarking". Benchmarking 10(3):176-209

38. Raithel J (2006) Quantitative Forschung: ein Praxiskurs, VS Verlag für Sozialwissenschaften, Wiesbaden

39. Fromm S (2006) Datenanalyse mit SPSS für Fortgeschrittene: Multivariate Verfahren für Querschnittsdaten, VS Verlag für Sozialwissenschaften, Wiesbaden

40. Bortz J (1999) Statistik für Sozialwissenschaftler: mit 247 Tab., Springer, Berlin (u.a.)

41. Field AP (2009) Discovering statistics using SPSS. SAGE, Los Angeles

42. Smith MJ, Sullivan JM, GN management (1997) Nurses' and patients' perceptions of most important caring behaviors in a long-term care setting. Geriatr Nurs 18(2):70-73

43. Wahl HW et al (2011) INSEL - Instrument zur praxisnahen Erfassung von Lebensqualität, Sindelfingen, Paul Wilhelm von Keppler-Stiftung

44. Benner P (2001) The phenomenon of care, in Handbook of Phenomenology and medicine. In: Toombs SK (Hrsg) Kluwer Academic Publisher, Dortrecht, S 351-370

45. Benner $P$ (2002) Caring for the silent patient. Am J Crit Care 11(5):480-481

46. Benner P (2003) Reflecting on what we care about. Am J Crit Care 12(2):165-166

47. Hawthorne G (2006) Measuring social isolation in older adults: development and Initial Validation of the friendship scale. Soc Indic Res 77(3):521-548

48. Mitchell PH et al (2003) A short social support measure for patients recovering from myocardial infarction: the enrichd social support inventory. J Cardiopulm Rehabil Prev 23(6):398-403

49. Attkisson CC, Greenfield TK (2004) The ucsf client satisfaction scales: I. The client satisfaction questionnaire-8, in The use of psychological testing for treatment planning and outcomes assessment: volume 3: instruments for adults 3. Aufl., Lawrence Erlbaum Associates Publishers, Mahwah S 799811

50. Jerusalem M, Schwarzer R (1999) SWE Skala zur Allgemeinen Selbstwirksamkeitsbeurteilung. Freie Universität Berlin, Berlin

51. Winkler I et al (2006) Der WHOQOL-OLD. Psychother Psych Med 56(EFirst):63-69

52. Bijl JVD, Poelgeest-Eeltink AV, Shortridge-Baggett $L$ (1999) The psychometric properties of the diabetes management self-efficacy scale for patients with type 2 diabetes mellitus. J Adv Nurs 30(2):352-359

53. Klann N, Hahlweg K, Heinrichs N (2003) Diagnostische Verfahren für die Beratung: Materialien zur Diagnostik und Therapie in Ehe-, Familien- und Lebensberatung, Hogrefe, Göttingen (u.a.), Verl. für Psychologie

54. Macera CA et al (1993) A measure of perceived burden among caregivers. Eval Health Prof 16(2):204-211

55. Brodbeck FC, Maier GW (2001) Das Teamklima-Inventar (TKI) für Innovation in Gruppen. Z. f. Arbeits- und Organisationspsychologie A\&O 45(2):59-73

56. Archbold PG et al (1990) Mutuality and preparedness as predictors of caregiver role strain. Res Nurs Health 13(6):375-384

\section{GBE veröffentlicht Themenheft „Rückenschmerzen"}

Ein aktuelles, kostenfrei erhältliches Themenheft der Gesundheitsberichterstattung (GBE) des Bundes befasst sich mit dem Thema

Rückenschmerzen. Insbesondere werden tiefsitzenden Rückenschmerzen thematisiert, die auch als Kreuzschmerzen bezeichnet werden. Die Ausgabe gibt einen Überblick zu Krankheitsbildern, Diagnostik, Risikofaktoren, Verlauf, Verbreitung, Folgen, Therapie, Versorgungssituation und Prävention.

Bei der Mehrzahl aller Rückenschmerz-Patienten lässt sich weder eine umschriebene Krankheit, noch ein krankhafter Prozess oder eine sichere anatomische Quelle als Ursache für den Schmerz finden. Bei diesen nicht-spezifischen Rückenschmerzen besteht eine komplexe Problematik. Risikofaktoren können aus sehr unterschiedlichen Quellen entstehen. Ein anerkanntes Risiko ist langjährige und schwere körperliche Arbeit. Eine überwiegend sitzende Tätigkeit scheint dagegen kein nennenswertes Risiko für Rückenschmerzen darzustellen. Auch die arbeitsbezogenen psychosozialen Bedingungen können eine Ursache für Rückenschmerzen sein. Darunter versteht man zum Beispiel eine geringe Arbeitsplatzzufriedenheit, monotone Arbeiten oder Konflikte. Personen mit einem niedrigen Sozialstatus berichten häufiger von Rückenschmerzen als Personen mit einem hohen Status. Zudem zeigt sich, je länger die gesamte Krankengeschichte eines Rückenschmerz-Patienten dauert, umso eher ist mit einem weiter ungünstigen Verlauf zu rechnen. Chronischen Rückenschmerzen, die länger als 3 Monate anhalten, sollten daher von den Betroffenen ernst genommen werden.

Für Diagnostik und Therapieplanung bei Rückenschmerzen kann das sog. Flaggenmodell hilfreich sein, das auf Begleitsymptome, Vorerkrankungen und psychosoziale Risikofaktoren aufmerksam macht. Eine Orientierung bei der Therapieplanung bietet die "Nationale VersorgungsLeitlinie Kreuzschmerz", von der es auch eine Patientenversion gibt (www. kreuzschmerz.versorgungsleitlinien.de). Invasive Therapien, Injektionen sowie operative Verfahren werden zum Beispiel nicht empfohlen. Körperliche Inaktivität und Schonung sollten vermieden werden, die Betroffenen sollten zur aktiven Mitwirkung an der Behandlung motiviert werden.
Das GBE-Heft „Rückenschmerzen“ kann kostenlos bestellt werden (E-Mail: gbe@rki.de, Fax: 030-18754-3513) und ist unter www.rki. de/gbe abrufbar.

Quelle: Robert Koch-Institut, Berlin, www.rki.de 